\title{
Reporte de caso: diverticulitis yeyunal perforada, causa infrecuente de abdomen agudo
}

\section{Case report: perforated jejunal diverticulitis, an infrequent cause of acute abdomen}

\author{
Guadalupe del C. Pérez-Torres*, Guadalupe K. Peña-Portillo, Sandra G. Ayala-Hernández, \\ Erwin I. Marín-Pardo y Édgar Grageda-Flores \\ Departamento Cirugía General, Institución Hospital Universitario de Puebla, BUAP, Puebla, Pue., México
}

\begin{abstract}
Resumen
Introducción: Los divertículos intestinales son infrecuentes, presentándose principalmente en el duodeno. Pocos pacientes desarrollan síntomas, siendo la perforación la que confiere mayor mortalidad. El tratamiento quirúrgico de urgencia en sospecha de divertículo yeyunal perforado se realiza en el 8-30\% de los casos, que comprende resección intestinal y anastomosis termino-terminal. Caso clínico: Paciente de sexo femenino de 101 años que se presenta al servicio de urgencias, con datos de abdomen agudo, se realiza tomografía de abdomen en donde se evidencia líquido libre y neumoperitoneo. Se decide realizar laparotomía exploradora, con evidencia de múltiples divertículos yeyunales, uno de ellos perforado, se realiza resección y anastomosis de $90 \mathrm{~cm}$ de yeyuno. Conclusión: A pesar de ser una patología poco frecuente, en pacientes con abdomen agudo el tratamiento de elección sigue siendo quirúrgico.
\end{abstract}

Palabras clave: Divertículo. Perforación intestinal. Abdomen agudo. Laparotomía.

\begin{abstract}
Introduction: Intestinal diverticula are infrequent, with most common presentation in duodenum. Only a few patients develop symptoms, being perforation the most mortal complication. 8-30\% of patients with suspicion of perforated jejunal diverticulum receive urgent surgical management, which consists in intestinal resection and termino-terminal anastomosis. Case report: 101 years old female patient who shows in emergency room with acute abdomen. Computed tomography is made, showing free liquid and pneumoperitoneum. Exploratory laparotomy is performed, with evidence of multiple jejunal diverticulum, one of them with perforation. We performed resection and anastomosis of $90 \mathrm{~cm}$ of the affected jejunum. Conclusion: Despite being an infrequent pathology, in patients with acute abdomen, the most accurate management remains surgery.
\end{abstract}

Key words: Diverticula. Intestinal perforation. Acute abdomen. Laparotomy.

Correspondencia:

*Guadalupe del C. Pérez-Torres

E-mail: drapereztorres@ hotmail.com
Fecha de recepción: 24-03-2021

Fecha de aceptación: 03-08-2021

DOI: 10.24875/RHJM.210000161
Disponible en internet: 17-12-2021 Rev Hosp Jua Mex. 2021;88(4):193-198

www.revistahospitaljuarez.com 1405-9622/@ 2021 Sociedad Médico-Quirúrgica del Hospital Juárez de México, A.C. Publicado por Permanyer. Este es un artículo open access bajo la licencia CC BY-NC-ND (http://creativecommons.org/licenses/by-nc-nd/4.0/). 


\section{Introducción}

Los divertículos intestinales son una entidad infrecuente, con una incidencia reportada en la literatura del 0.1 al $2.6 \%{ }^{1-5}$ y hasta el $4.6 \%^{6}$, correspondiendo el $80 \%$ al yeyuno proximal, el $15 \%$ al íleon (de los cuales un $75 \%$ en yeyuno proximal, un $20 \%$ en distal y un $5 \%$ en íleon) $)^{7,8}$ y el $5 \%$ a ambos ${ }^{9-11}$. Son herniaciones de la pared intestinal y pueden ser verdaderos o falsos según la presencia o no de todas las capas intestinales, respectivamente. Tienen una prevalencia mayor en los hombres y pacientes de la tercera edad. La presentación suele ser asintomática, pero en algunos casos pueden presentar síntomas gastrointestinales inespecíficos. En un menor porcentaje pueden presentar complicaciones como sangrado, obstrucción y perforación, con una mortalidad de hasta el $40 \%{ }^{12-14}$. El diagnóstico prequirúrgico continúa siendo un reto debido a la dificultad para identificarlos en los estudios de imagen, o la inaccesibilidad a los más sensibles. Por ello, la terapéutica más empleada hasta el momento es quirúrgica, aunque estudios recientes han demostrado la efectividad del manejo conservador. Presentamos el caso de una paciente de 101 años que acudió a nuestro servicio de urgencias con un cuadro de abdomen agudo, resultando en la perforación de un divertículo yeyunal.

\section{Métodos. Caso clínico}

\section{Presentación de caso}

Mujer de 101 años, con antecedentes personales de infarto agudo de miocardio, apendicectomía y colecistectomía abiertas, y plastia de pared. Niega sintomatología gastrointestinal previa. Inicia su padecimiento actual cuatro días previos a su valoración, con dolor cólico en mesogastrio de intensidad moderada, con aumento progresivo hasta ser generalizado, acompañado de náuseas. Se automedica con diclofenaco y ácido acetilsalicílico, no especifica posología, sin mejoría de sintomatología, agregándose cefalea y evacuaciones disminuidas en consistencia en tres ocasiones, sin moco ni sangre. A la exploración física refiere dolor generalizado grave, escalofríos, astenia y adinamia. Presenta hipotensión arterial y taquicardia. El abdomen con distensión abdominal, peristalsis disminuida, rigidez muscular, doloroso a la palpación, signo de von Blumberg presente.

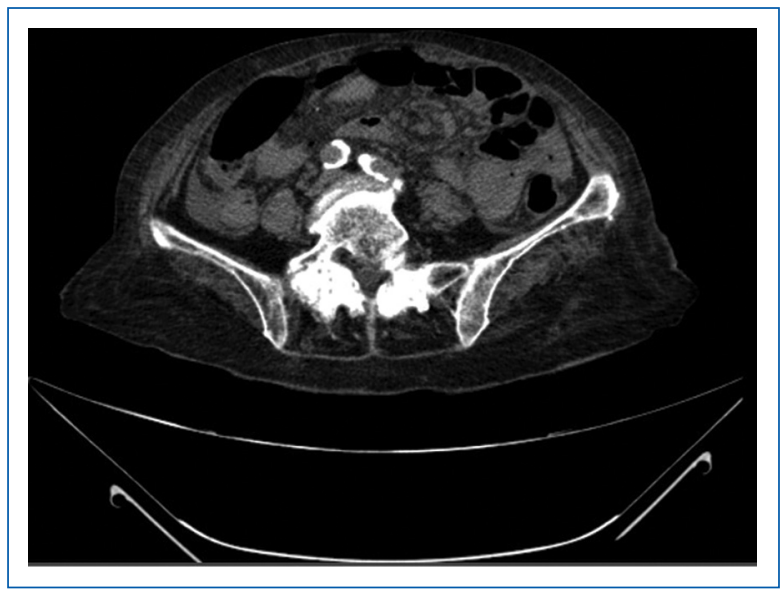

Figura 1. Tomografía simple de abdomen, donde se observa líquido libre en corredera parietocólica y neumoperitoneo.

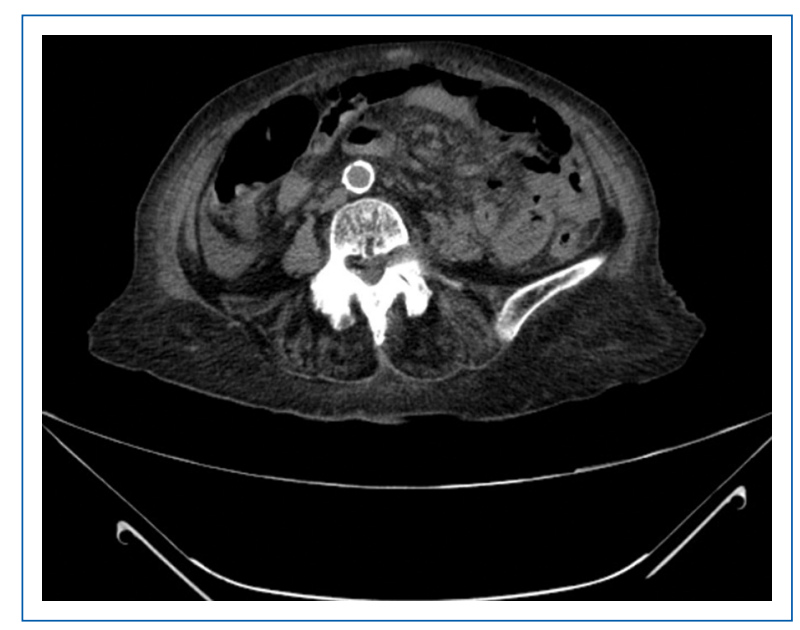

Figura 2. Tomografía simple de abdomen, corte axial, que reporta signo de "remolino", dato clínico de oclusión intestinal alta o de malrotación intestinal.

\section{Investigaciones}

Los estudios de laboratorio reportan leucopenia (3.07 $\times 109 \mathrm{I})$, neutrofilia (84\%), hiperprocalcitoninemia (2.29 ng/ml), acidosis metabólica $(\mathrm{pH}: 7.31$, presión parcial de dióxido de carbono: $27.9 \mathrm{mmHg}$, bicarbonato: $15.8 \mathrm{mmHg})$ e hiperlactatemia $(5.90 \mathrm{mmol} / \mathrm{l})$; resto dentro de parámetros normales.

Se realiza tomografía simple de abdomen donde se reporta neumobilia, neumoperitoneo, zona de transición asociada a "signo de remolino" en topografía de yeyuno, en asa cerrada (Figs. 1-2); datos compatibles con obstrucción de intestino 6 delgado de alto grado, con líquido libre en corredera parietocólica derecha 


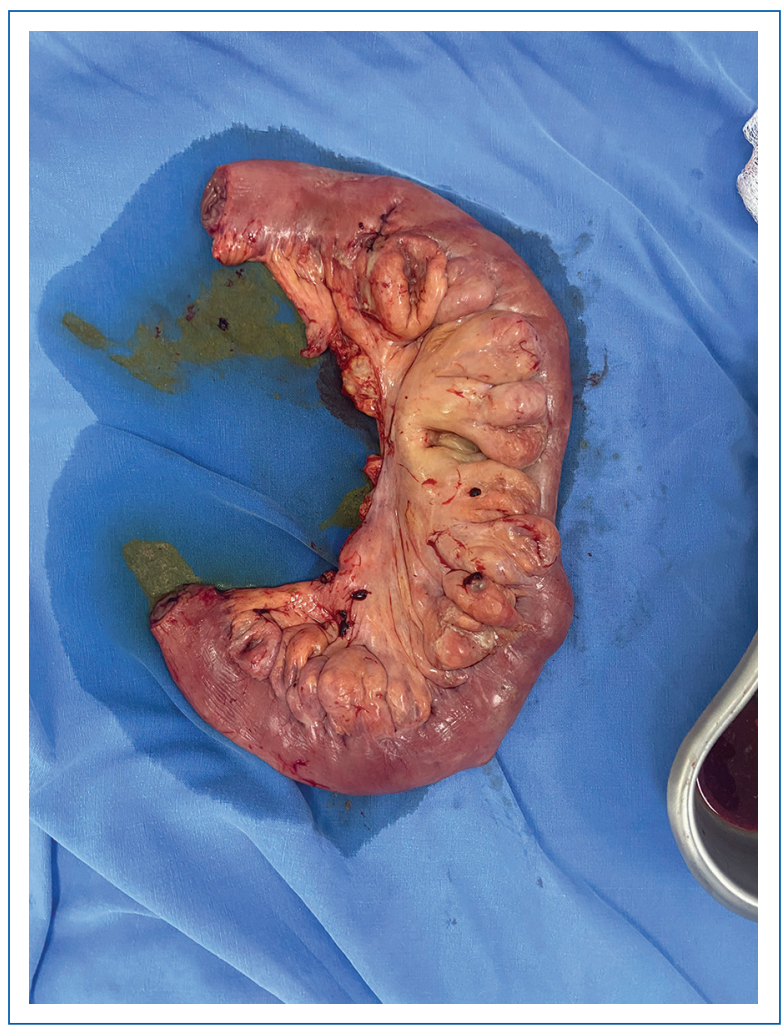

Figura 3. Pieza de patología, $40 \mathrm{~cm}$ de yeyuno, con Divertículo yeyunal perforado, con material de sutura.

(Fig. 1). Se integra el diagnóstico de abdomen agudo secundario a perforación de víscera hueca.

\section{Resultados}

Se realiza laparotomía exploradora, donde se identifican $1,000 \mathrm{ml}$ de líquido intestinal libre en cavidad, asas intestinales con placas de fibrina, perforación de $1 \mathrm{~cm}$ en divertículo yeyunal de aproximadamente $6 \times 4 \mathrm{~cm}$, a $50 \mathrm{~cm}$ del ángulo de Treitz, con múltiples divertículos en aproximadamente $80 \mathrm{~cm}$ de yeyuno posterior a la perforación, se realiza inicialmente cierre primario y posteriormente resección intestinal de aproximadamente $90 \mathrm{~cm}$, con yeyuno-yeyuno anastomosis término-terminal en dos planos.

Egresa del quirófano a terapia intermedia, donde se brinda manejo y egresa al servicio de cirugía general a los dos días por mejoría, iniciando nutrición parenteral. A los tres días inicia la vía oral con dieta líquida y progresa al día siguiente a dieta en papilla, tolerándola adecuadamente, por lo que se suspende la nutrición parenteral. Es egresada a los siete días de la cirugía y valorada en consulta externa 12 días después, presentando

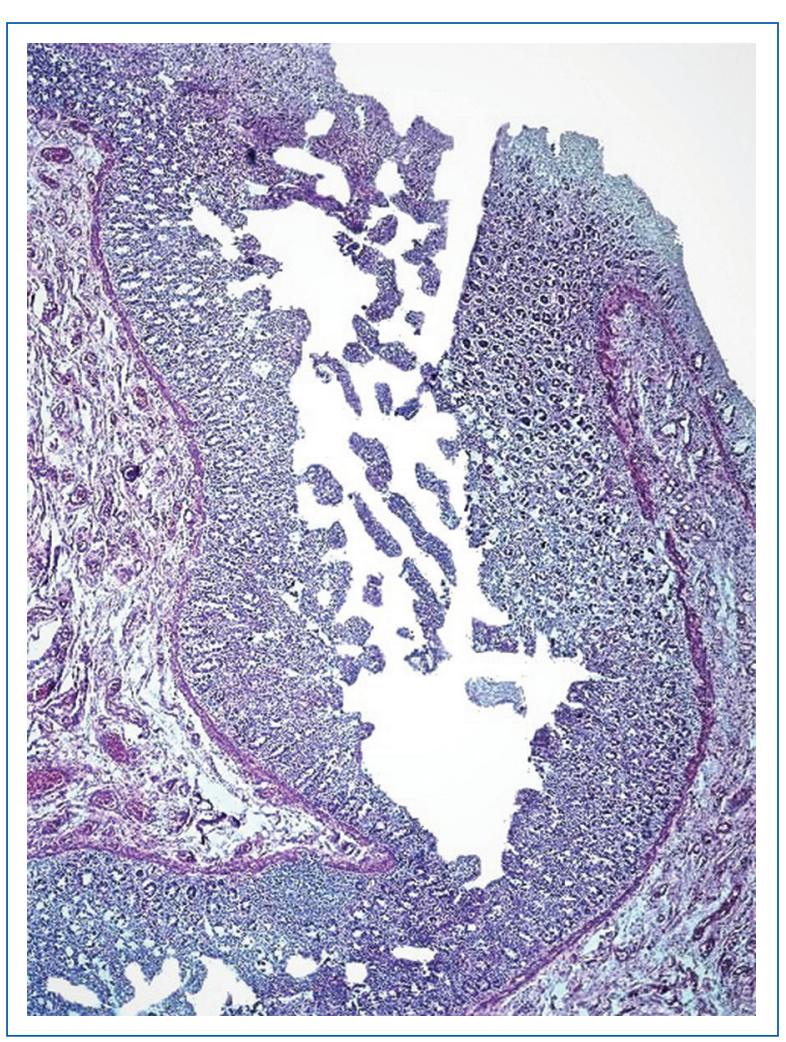

Figura 4. Se observa la mucosa invaginada entre la pared, formando el divertículo.

adecuada evolución. Se recaba reporte histopatológico con segmento de intestino de $40 \mathrm{~cm}$ de longitud y $3.2 \mathrm{~cm}$ de circunferencia, con adherencias de material fibrinoso en mesenterio, con saculaciones, una de ellas con material de sutura que se localiza a $7 \mathrm{~cm}$ de uno de los límites quirúrgicos (Fig. 3), presentando pared delgada con congestión, con enfermedad diverticular perforada que condicionó peritonitis aguda (Figs. 4-7).

\section{Discusión}

Un divertículo intestinal es una protrusión sacular que suele ser adquirida de la pared intestinal en los sitios de mayor debilidad (donde penetran los vasos sanguíneos), que involucra únicamente las capas mucosa y submucosa, siendo un pseudodivertículo $0^{6,8,11-13}$. En el intestino delgado se observan con mayor frecuencia en el duodeno $(80 \%)$, presentándose hasta en el $10-20 \%$ de las personas ${ }^{15}$, seguido del yeyuno e íleon $(20 \%)^{16}$, presente en el $1 \%$ de la población ${ }^{17}$, donde suelen ser múltiples, con localización yeyunal y cercanos al ángulo de Treitz $^{18}$. Esta formación en los sitios de debilidad explica su localización en el borde mesentérico ${ }^{18-20}$, mientras 


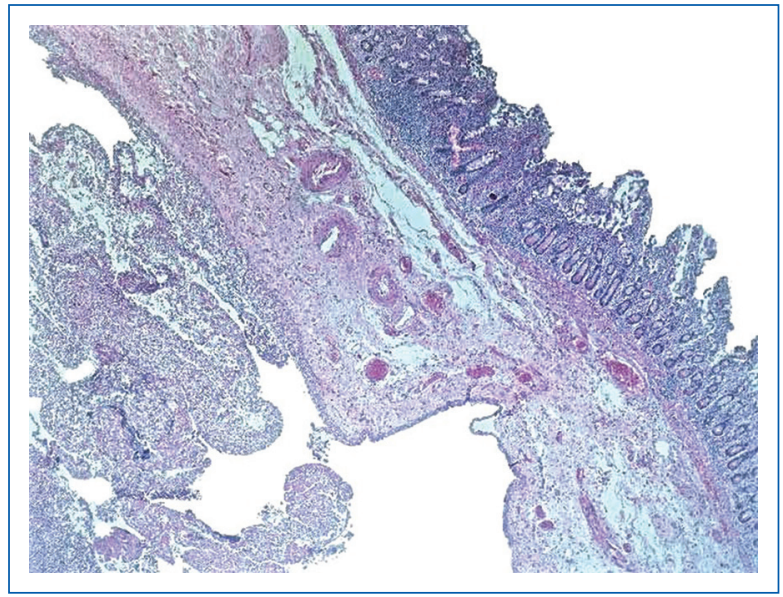

Figura 5. Se observa divertículo yeyunal, sin pared muscular, únicamente mucosa.

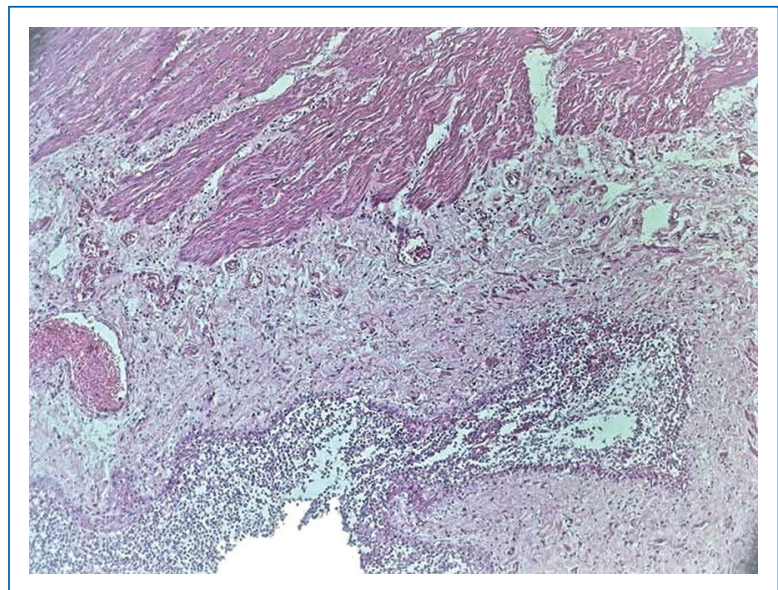

Figura 6. Se observa la pared muscular, subserosa, serosa: cubierta de neutrófilos y fibrina, que indica proceso inflamatorio causando peritonitis.

que los de localización ileal suelen ser únicos y de menor tamaño ${ }^{21,22}$. Esto se explica por el mayor diámetro de los vasos sanguíneos en el yeyuno proximal ${ }^{23,24}$. Suelen estar acompañados de divertículos en otros sitios hasta en un $90 \%{ }^{21,22}$, como en colon (30-75\%), duodeno (15-42\%), vejiga (12\%) y esófago $(2 \%)^{20,21,25}$, por ello se deben buscar de forma intencional al observarlos en el transoperatorio. Predominan en el sexo masculino (1.5:1) y se observan con mayor frecuencia entre la sexta y séptima décadas de la vida ${ }^{3,6,8-11,13,14}$.

Un $10-40 \%$ puede llegar a presentar complicaciones ${ }^{1,2,8,14}$, siendo la diverticulitis la más frecuente $(2-6 \%)^{6}$, seguida de hemorragia $(5-33 \%)$ y obstrucción intestinal (2.3-4.6\%) $)^{2,3}$. Puede haber perforación

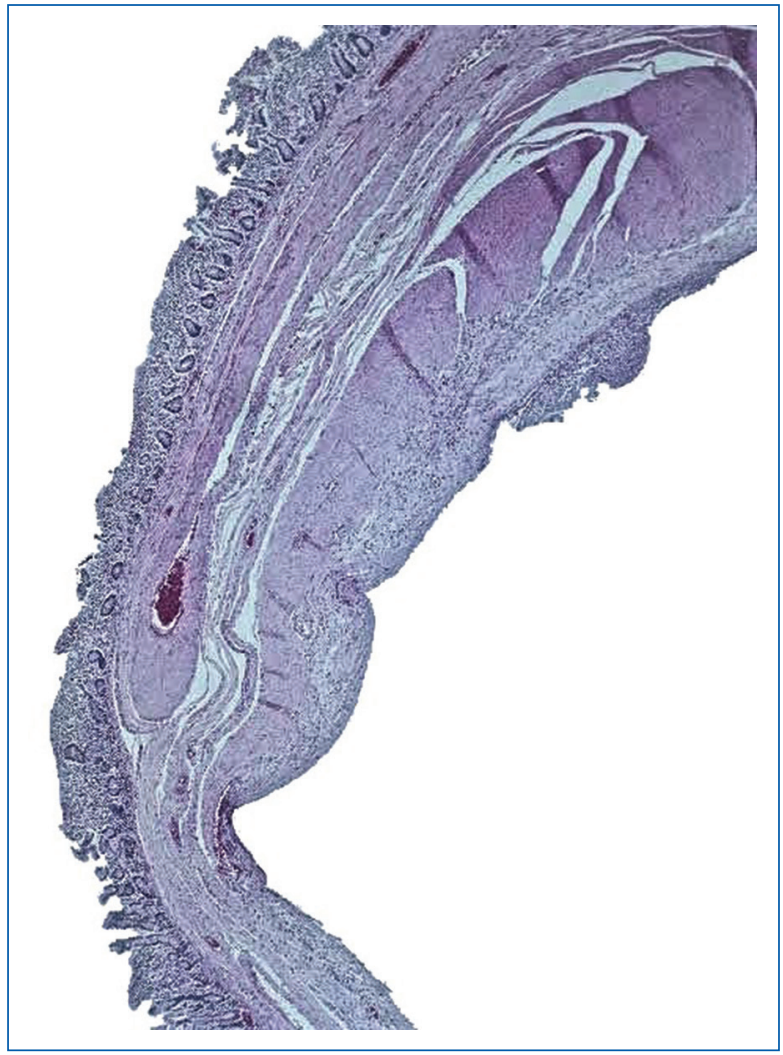

Figura 7. Se observa, parte del divertículo yeyunal con disminución de su pared muscular, causado por peritonitis.

en un 2-7\% $\%^{2,10}$. Otras complicaciones descritas son vólvulos $9,{ }^{15}$, obstrucción, abscesos hepáticos, neumoperitoneo espontáneo, esteatorrea 0 anemia megaloblástica ${ }^{14}$. Pueden encontrarse abscesos en la raíz del mesenterio, secundarios a la perforación de los divertículos ubicados en esta zona ${ }^{14}$. La presentación clínica de la perforación puede ser con peritonitis localizada 0 generalizada ${ }^{21}$. Las causas de perforación se han asociado con reacción inflamatoria necrotizante (82\%), impactación de un cuerpo extraño (6\%) y trauma cerrado de abdomen $(12 \%)^{1,6,7,14}$. La mortalidad de la perforación asciende hasta el $40 \%{ }^{1-3}$. Algunos factores de mal pronóstico son edad avanzada, presencia de comorbilidades, peritonitis, retraso en el diagnóstico y el manejo terapéutico ${ }^{2}$. La presentación clínica suele cursar con dolor abdominal localizado (39\% en flanco izquierdo), rigidez abdominal, fiebre, leucocitosis y elevación de la proteína $\mathrm{C}$ reactiva ${ }^{2}$. La obstrucción intestinal es la complicación menos frecuente (2.3-4.6\%) y se relaciona con otros mecanismos, como compresión extrínseca por un pseudotumor secundario a diverticulitis; un vólvulo debido a adherencias posteriores a cuadros repetitivos de diverticulitis pauci-sintomática; y 
estenosis fibrosa del intestino después de uno o más cuadros de diverticulitis ${ }^{6}$. Un reporte describe la asociación a un fecalito gigante ${ }^{20}$. El tratamiento conservador no ha demostrado utilidad, por lo que el manejo es quirúrgico con resección intestinal y de la adherencia involucrada, en caso de estenosis, necrosis o perforación ${ }^{6}$. La hemorragia diverticular (2-8.1\%) puede ocasionarse por la propia diverticulitis o por una úlcera diverticular, enterolito o ingesta de antiinflamatorios no esteroideos $^{6,21,22}$. El tratamiento suele ser quirúrgico, pero existen reportes que describen el manejo conservador con resultados exitosos 22,23 . Se han descrito otras complicaciones menos frecuentes, como resección hepática secundaria a la adherencia del divertículo al borde hepático y presencia de tejido pancreático ectópico en su interior ${ }^{24,25}$.

El tratamiento quirúrgico de urgencia se realiza en el 8-30\% de los pacientes con diverticulosis yeyunal complicada ${ }^{6}$. Está indicado en caso de falla o imposibilidad de un drenaje percutáneo, y en peritonitis generalizada $^{6}$. Comprende resección intestinal del segmento afectado y anastomosis primaria término-terminal, y drenajes percutáneos radioguiados en caso de colecciones localizadas $1,2,5-8,10,12-14,17,19,26,27$. Esta resección también es obligatoria en pacientes con un divertículo de gran tamaño y asas intestinales dilatadas e hipertróficas, debido al riesgo de complicaciones ${ }^{6}$. Se puede valorar también realización de estoma en pacientes con inestabilidad hemodinámica 0 de alto riesgo $0^{6}$. En caso de diverticulosis extensa se debe limitar la resección al segmento con el divertículo perforado, para evitar un síndrome de intestino corto 6,15,18. Las técnicas de invaginación o escisión local están contraindicadas debido a la tasa elevada de morbimortalidad con estos métodos ${ }^{1,4,6-8}$. El abordaje continúa siendo mediante laparotomía exploradora. Existen casos que reportan la utilidad del abordaje laparoscópico, sin embargo, su uso no está establecido debido a la falta de experiencia por la infrecuencia de esta patología ${ }^{28}$. La tasa de mortalidad del tratamiento quirúrgico es del $0-5 \%$. Algunos factores de mal pronóstico son edad avanzada, comorbilidades asociadas, peritonitis, retraso en el diagnóstico (40\%) y el tiempo entre la perforación y la cirugía 6 .

\section{Conclusión}

Los divertículos intestinales son poco frecuentes, cursando de forma asintomática en la mayoría de los casos, sin embargo, aquellos pacientes que desarrollan sintomatología pueden cursar con clínica poco específica. Aquellos con sospecha de complicaciones deben ser evaluados de forma integral. En el caso clínico que se describe la paciente ingresa a urgencias hemodinámicamente estable, con datos de respuesta inflamatoria sistémica y abdomen agudo, cumpliendo con el grupo etario más frecuente de presentación. Durante la anamnesis la paciente niega sintomatología gastrointestinal previa, iniciando con perforación del divertículo intestinal. El tratamiento quirúrgico fue el abordaje de elección, asociado a resección intestinal y no solo diverticulectomía, ya que se ha evidenciado peor pronóstico, presentando evolución clínica favorable a pesar de la edad.

\section{Agradecimientos}

Los autores agradecen a los médicos adscritos al servicio de Cirugía General del Hospital Universitario de Puebla por la enseñanza brindada para la realización de este trabajo, especialmente a la jefa del servicio y a su titular, por su apoyo incondicional. Así también, los autores hacen especial mención a la médica pasante del servicio social, María Antonia Jiménez Durán, por proveer las imágenes utilizadas.

\section{Conflicto de intereses}

Los autores declaran no tener conflicto de intereses.

\section{Financiamiento}

Los autores declaran que la presente investigación no ha recibido ninguna beca especifica de agencias de sectores públicos, comercial o sin animo de lucro.

\section{Responsabilidades éticas}

Protección de personas y animales. Los autores declaran que para esta investigación no se han realizado experimentos en seres humanos ni en animales.

Confidencialidad de los datos. Los autores declaran que han seguido los protocolos de su centro de trabajo sobre la publicación de datos de pacientes.

Derecho a la privacidad y consentimiento informado. Los autores han obtenido el consentimiento informado de los pacientes y/o sujetos referidos en el articulo. 


\section{Bibliografía}

1. Alves Martins BA, Rodrigues Galletti R, Marinho Dos Santos Neto J, Neiva Mendes C. A case of perforated jejunal diverticulum: An unexpected cause of pneumoperitoneum in a patient presenting with an acute abdomen. Am J Case Rep. 2018;19:549-52.

2. Lebert $P$, Ernst $O$, Zins $M$. Acquired diverticular disease of the jejunum and ileum: imaging features and pitfalls. Abdom Radiol. 2019;44(5):1734-43.

3. Kumar D, Meenakshi. Complicated jejunal diverticulitis with unusual presentation. Radiol Case Rep. 2017;13(1):58-64.

4. Luján D, Ruiz M, Peña E, Pastor P, Candel MF, Albarracín A. Diverticulitis yeyunal perforada. Una causa infrecuente de abdomen agudo grave. REIQ. 2017;20:119-20.

5. Lebert P, Millet I, Ernst O, Boulay-Coletta I, Corno L, Taourel P, et al Acute jejunoileal diverticulitis: multicenter descriptive study of 33 patients. AJR Am J Roentgenol. 2018:210(6):1245-51.

6. Harbi H, Kardoun N, Fendri S, Dammak N, Toumi N, Guirat A, et al Jejunal diverticulitis. Review and treatment algorithm. Presse Med 2017;46(12 Pt 1):1139-43.

7. Vásquez MA, Fuerte Ruiz S, León Ledesma R, Jover Navalón JM. Abdomen agudo secundario a perforación de divertículo yeyunal. Span $J$ Surg Res. 2016;19(2):71-2.

8. Sehgal R, Cheung CX, Hills T, Waris A, Healy D, Khan T. Perforated jejunal diverticulum: a rare case of acute abdomen. J Surg Case Rep. 2016;2016(10):rjw169.

9. Gallego Mariño A, Ramírez Batista A, Amado Martínez JA. Divertículos de intestino delgado. Revista Electrónica Dr. Zoilo E. Marinello Vidaurreta. $2016 ; 41(4)$.

10. López Marcano AJ, Ramia JM, de la Plaza Llamas J, Alonso S, Gonzales Aguilar JD, Kühnhardt Barrantes AW. Enfermedad diverticular yeyuno-ileal complicada tratada quirúrgicamente: serie de 12 casos y revisión de literatura. Rev Gastroenterol Peru. 2017;37(3):240-5.

11. Mohi RS, Moudgil A, Bhatia SK, Seth K, Kaur T. Complicated jejunal diverticulosis: Small bowel volvulus with obstruction. Iran J Med Sci. 2016;41(6):548-51.

12. Serrano-González J, Artés-Caselles M, Román-García de León L, Pla-Sánchez P, Sánchez-Turrión V. Manejo de la diverticulitis yeyunal. Experiencia en nuestro centro. Cir Cir. 2018;86(2):148-51.

13. Dudric VN, Axente DD. Perforated jejunal diverticulum, a case report. HVM Bioflux. 2018;10(4):181-3.
14. Calcerrada E, Mirón B, Triguero J, Lendínez I, Sarabia N, Torres Alcalá T. Perforación de divertículo yeyunal como causa de abdomen agudo. RMP. 2016;36(3):34-7.

15. Aradhya Nigam, Faye F. Gao, Mark A. Steves, Paul H. Sugarbaker. Acute abdomen caused by a large solitary jejunal diverticulum that induced a midgut volvulus. Report of a case. IntJ Surg Case Rep. 2020;74:109-12.

16. Hernández R, Pontillo $M$, Rodríguez $G$. Divertículo yeyunal perforado: causa excepcional de abdomen agudo. Cir Urug. 2018;2(1):27-31.

17. Yeung E, Kumar V, Dewar Z, Behm R. Rare aetioloy of abdominal pain: contained abscess secondary to perforated jejunal diverticulitis. BMJ Case Rep. 2020;13:e235974.

18. Jambulingam R, Nanayakkara G. Non-operatively managed case of contained jejunal diverticular perforation. BMJ Case Rep 2019;12:e228811.

19. Sai Suraj K, Chaithanya J, Hariprasad TR, Rajagopalan S. Large jejuna diverticular faecolith causing small bowel obstruction: a bizarre cause of an acute abdomen. Int Surg J. 2019;6(10):3863-5.

20. Curcio G, Ligresti D, Ricotta C, Gruttadauria S, Traina M. Massive bleeding from a jejunal diverticulum reached and treated by underwater single-balloon enteroscopy. Gastrointest Endosc. 2016;84(6):1068-9.

21. Shoji M, Yoshimitsu Y, Maeda T, Sakuma H, Nakai M, Ueda H. Perforated gastrointestinal stromal tumor (GIST) in a true jejunal diverticulum in adulthood: report of a case. Surg Today. 2014;44(11):2180-6.

22. Rankothkumbura JP, Bogammana GMK. Perforated non Meckelian jejunal diverticulum: a rare cause of acute abdomen. Sri Lanka Journal of Surgery. 2018:36(4):38-9.

23. Horesh N, Klang E, Gravetz A, Nevo Y, Amiel I, Amitai MM, et al. Jejunal diverticulitis. J Laparoendosc Adv Surg Tech A. 2016;26(8):596-9.

24. Koli D, Vats $M$, Vardhan-Upreti H. Perforated isolated jejunal diverticular: a rare cause of acute abdomen. Clin J Gastroenterol. 2020;13(5):728-31. Retraction Note: Clin J Gastroenterol. 2021;14(2):697.

25. De la Ossa J, Kachedrian T, Panicker L, Sobrado J, Mohan K. A case of jejunal diverticulum causing life-threatening hemorrhage. Am J Gastroenterol. 2019;114:S1106-S1108.

26. Pushpa, Desai CJ, Kulhari M. Giant jejunal diverticulum required liver wedge resection: a rare case report. Indian J Surg. 2020;83:1023-5.

27. Shiratori H, Nishikawa T, Shintani Y, Murono K, Sasaki K, Yasuda K, et al. Perforation of jejunal diverticulum with ectopic pancreas. Clin $J$ Gastroenterol. 2017;10:137-41.

28. Buils Vilalta FJ, Sánchez Cano JJ, Domènech J, Prieto Butillé R,Homs FerréE, et al. Laparoscopic treatment of a patient with perforated jejunal diverticulitis. J Med Res Surg. 2020;1(3):1-4. 